\title{
Introducing and Integrating Perinatal Mental Health Screening: Development of an Equity-informed Evidence-based Approach
}

\section{Rebecca Blackmore}

Monash University Faculty of Medicine Nursing and Health Sciences https://orcid.org/0000-00024617-0609

Jacqueline A Boyle

Monash University Faculty of Medicine Nursing and Health Sciences

Kylie M Gray

University of Warwick

Suzanne Willey

Monash University

Nicole Highet

Centre of Perinatal Excellence

Melanie Gibson-Helm ( $\nabla$ melanie.gibson@monash.edu )

Monash University Faculty of Medicine Nursing and Health Sciences

\section{Research}

Keywords: perinatal, mental health, depression, anxiety, refugee, implementation science

Posted Date: June 17th, 2021

DOI: https://doi.org/10.21203/rs.3.rs-593027/v1

License: (c) (1) This work is licensed under a Creative Commons Attribution 4.0 International License. Read Full License 


\section{Abstract}

Background: Pregnancy is a time of increased risk for developing or re-experiencing mental illness. Perinatal mental health screening for all women is recommended in many national guidelines but a number of systems-level and individual barriers often hinder policy implementation. These barriers result in missed opportunities for detection and early intervention, and are likely to be experienced disproportionately by women from culturally and linguistically diverse backgrounds, including women of refugee backgrounds. The objectives of this study were to develop a theory-informed, evidence-based guide for introducing and integrating perinatal mental health screening across health settings; and to synthesise the learnings from an implementation initiative and multi-sectoral partnership between the Centre of Perinatal Excellence (COPE), and a university-based research centre. COPE is a peak body in perinatal mental health and Non-Governmental Organization commissioned to update the Australian national perinatal mental health guidelines, train health professionals and rollout innovative digital screening across healthcare settings.

Methods: In this case study, barriers to implementation were prospectively identified and strategies to overcome them were developed. A pilot perinatal screening program with a strong health equity focus was implemented and evaluated at a large public maternity service delivering care to a culturally diverse population of women in metropolitan Melbourne, Australia, including women of refugee background. Strategies identified pre-implementation and post-evaluation were mapped to theoretical frameworks. An implementation guide was developed to support future policy, planning and decision-making by healthcare organisations.

Results: Using a behavioural change framework (COM-B), the key barriers, processes, and outcomes are described for a real-world example designed to maximise accessibility, feasibility, and acceptability. A program logic model was developed to demonstrate the relationships of the inputs, which included stakeholder consultation, resource development, and a digital screening platform, with the outcomes of the program. A seven-stage implementation guide is presented for use in a range of healthcare settings.

Conclusions: These findings describe an equity-informed, evidence-based approach that can be used by healthcare organisations to address common systems and individual level barriers to implement perinatal mental health screening guidelines.

\section{Contributions To The Literature}

- These results present strategies that were informed by prior research and developed iteratively to address the identified barriers to implementing perinatal mental health screening.

- To the best of our knowledge, this is the first study to apply the Capability, Opportunity, Motivation Behaviour Model (COM-B) to the implementation of a perinatal mental health screening program, which allowed for a greater understanding of how the delivered strategies resulted in behaviour change. 
- These findings provide a road map of strategies based on implementation science along with an implementation guide to assist with the integration of perinatal mental health screening across many settings.

\section{Background}

Perinatal mental health has been acknowledged by the World Health Organization as a significant public health issue directly impacting maternal morbidity, obstetric outcomes, as well as infant attachment and development [1-4]. Pregnancy is a critical time in regards to mental health as there is an increased chance of women experiencing or re-experiencing mental illness [5-7]. Associated long-term costs as a result of perinatal mental illness, including costs to healthcare, wellbeing, productivity, and intergenerational impact, have been assessed at $\$ 5.2$ billion in Australia [8] and $£ 8.1$ billion pounds per year in the UK [9].

During the perinatal period, defined as conception to twelve months following birth, depression and anxiety are the most commonly experienced mental illnesses [10,11]. For women living in high-income countries, perinatal depression and anxiety is reported to affect up to $13.0 \%[12,13]$. For women living in low-and-middle-income countries, the prevalence of perinatal depression is more than double this rate with a pooled prevalence of $31 \%$ for any depressive illness [14]. In particular, women of refugee background are at an even greater risk of mental illness during pregnancy. This is attributable to the conflict, trauma, separation from family, and protracted situations of uncertainty which are hallmarks of refugee experiences.

Screening for mental illness during pregnancy is justified to improve health outcomes for women and their children. Participation in perinatal depression screening programs has been shown to improve identification of women at risk, referral uptake, and engagement with services, which in turn has a positive impact on mental health outcomes [15]. Routine, standardised screening in pregnancy for mental illness is recommended in high income countries including the United Kingdom [16], the United States of America [17] and Australia [5]. However, this is a common evidence-practice gap, with screening poorly implemented due to a lack of knowledge and support for women and health professionals. Additionally, there are a number of systems-level and individual barriers including short consultation times, inadequate funding, absence of clear referral pathways, and insufficient mental health training [18]. This represents a critical gap in best-practice pregnancy care resulting in the under-recognition of women at risk of perinatal mental illness.

In order to ensure screening is successful and improves health care, the barriers to implementation must be addressed for all women, including those with complex care needs or who commonly experience additional barriers to accessing best-practice care, such as women of refugee background. Additional challenges to perinatal mental health screening when working with refugee populations can include availability of face-to-face interpreters, health literacy, and cultural barriers such as the stigma of disclosing symptoms of mental illness [18]. 
Implementing sustainable change within healthcare settings presents a number of complex challenges. The aim of this paper is to provide a summary of strategies devised and delivered to address the complex nature of implementation. A perinatal mental health screening program using a digital screening program implemented at a large public maternity service in metropolitan Melbourne is presented as the exemplar, with additional relevance to services with multicultural populations, or populations with the potential for complex risk profiles. Barriers and enablers to implementation were identified during formative research and stakeholder consultation preceding initial design of the program [18]. Further strategies were developed iteratively during implementation to address additional barriers as they arose. An evaluation of the program yielded further refinements for large-scale, sustainable roll-out. Here, we bring the learnings from each stage and interpret them together for the first time. This novel synthesis uses the Theoretical Domains Framework (TDF) [19], a Program Logic Model [20], and the Capability, Opportunity, Motivation

- Behaviour Model (COM-B) [21] to further understand the barriers and required interventions for successful behaviour change. Concurrent with the implementation and evaluation of this program, a perinatal mental health screening implementation guide was developed to further assist health services implement perinatal mental health guidelines in their specific context.

\section{Methods}

The Standards for Reporting Implementation Studies (StaRI) [22] checklist was used to report this study.

\section{Setting}

We used a case study approach [23] to describe how the perinatal mental health screening program was designed and implemented. The protocol for the evaluation of this program has been published elsewhere [24], but in summary, the study was conducted at a public antenatal clinic located in a large teaching hospital within south-eastern Melbourne, Australia. The area is home to a large multicultural and refugee population. This clinic was designed to specifically cater for women of refugee background one day per week, supported by a refugee health nurse liaison and two bi-cultural workers. Approximately half of the women who attended this clinic were of refugee background or considered to be refugee-like, i.e. arrived in Australia on a spousal visa from a refugee-source country including Afghanistan, Myanmar, the Republic of South Sudan, and Sri Lanka.

\section{Implementation Strategy Development}

The development of the implementation strategies were informed by the findings of the formative research with stakeholders. This involved semi-structured interviews with health professionals $(n=28)$ such as midwives, obstetricians, perinatal mental health and refugee health experts, interpreters as well as women of refugee background $(n=9)$ [18]. The results from this research identified the barriers and enablers within eight key Theoretical Domains Framework (TDF) domain constructs [19]. A steering committee was then formed to facilitate implementation. The committee comprised of staff from key hospital departments, GP liaison, refugee health and wellbeing, the Non-Governmental Organization (NGO) COPE and academic experts in psychology, midwifery, obstetrics and public health. This 
committee met fortnightly for two years to devise strategies to address the barriers, implement and evaluate the program. The committee addressed concerns of the research team or hospital staff as they arose and responded with practical solutions. In addition, a community advisory group was also formed and comprised of women from eight different countries, some of refugee backgrounds, with most having recently had a baby at the health service. This committee met bi-monthly and was instrumental in planning the implementation and evaluation such as recruitment strategies, resources, and facilitating an understanding of the cultural complexity of the women participating in the study.

The acceptability and feasibility of the perinatal mental health screening program in the antenatal period was evaluated from the both the perspective of the health professionals as well as women of refugee background and these results have been published elsewhere $[25,26]$ In summary, a mixed methods approach was undertaken whereby health professionals completed an online survey $(n=38)$, focus groups ( $n=2,13$ participants), and semi-structured interviews $(n=8,11$ participants). The evaluation was guided by the Normalization Process Theory (NPT) [27]. The perspectives of women of refugee background was evaluated using focus groups ( $n=1 ; 5$ participants) and semi-structured telephone interviews $(n=17)$. The evaluation findings led to the development of strategies required for program refinement and scale-up. In the synthesis presented here, these strategies are described and interpreted using implementation theories and behaviour change frameworks for the first time.

\section{Synthesis Process}

Three frameworks were used to better understand the relationships between barriers and strategies, inputs, and outcomes associated with implementing perinatal mental health screening.

\section{Theoretical Domains Framework (TDF)}

The TDF is an integrated framework of theoretical constructs that are related to behaviour change (19). The TDF was used to map the strategies and barriers devised before, during, and after implementation.

\section{Program Logic Model}

A program logic model was developed to demonstrate the relationships of the inputs, outputs, and outcomes of the perinatal mental health screening program [20]. Logic models are useful in evaluating how outcomes are produced by processes (inputs), however a logic model alone is not sufficient enough to capture the dynamics of complex interventions [28]. Therefore, the theoretical framework of the COM-B model was applied to understand the requirements for behaviour change.

3. The Capability, Opportunity, Motivation - Behaviour Model (COM-B)

The Capability, Opportunity, Motivation, Behaviour (COM-B) model is part of the Behaviour Change Wheel (BCW) [21]. It proposes that for someone to engage in a behaviour, they must be physically and psychologically able (capability), have the social and physical opportunity to do the behaviour (opportunity), and want to do the behaviour more than other competing behaviours (automatic and 
reflective motivation) [21]. As each of the TDF domains relates to a component of the COM-B model (Figure 1 and Table 1), when incorporated together this provides greater understanding of behaviour change to improve implementation.

\section{Developing an Implementation Guide: Translation of Evidence into Practice}

The Centre of Perinatal Excellence (COPE) was represented on the steering committee from the screening program's inception. COPE is a peak body in perinatal mental health and the Non-Governmental Organization commissioned to update the Australian national perinatal mental health guidelines and rollout innovative digital screening across healthcare settings. Based on the learnings from this pilot screening program, as well as implementation of the digital platform across other healthcare settings, COPE has developed a guide to assist healthcare services in implementing perinatal mental health screening.

\section{Results}

Some barriers and enablers to perinatal mental health screening were identified prior to implementation and mapped to the TDF previously [18]. Here we build on the pre-implementation mapping to describe and interpret barriers identified at all three stages and the strategies devised to address said barriers, using the TDF domains: knowledge and skills, social/professional role and identity, beliefs about capabilities and consequences, environmental context and resources, social influences, and behavioural regulation.

\section{Identified Barriers and Strategies for Implementation}

\section{Knowledge and Skills}

Pre-implementation barriers

Health professionals acknowledged the importance of routine antenatal mental health screening. However, a lack of knowledge and skills regarding mental health screening measures, administration and scoring, and the specific health needs of women of refugee background was identified as a barrier to screening [18].

\section{Pre-implementation strategies}

Training for all staff in the antenatal clinic, including midwives, obstetricians, and administrative staff, was delivered as part of existing regular staff meetings within the organisation. In attendance, members of the research team; a midwife and two psychologists, provided training on perinatal depression and anxiety, suicide risk assessment, administration and scoring of the recommended screening measure the Edinburgh Postnatal Depression Scale (EPDS) [30], use of the digital platform iCOPE [31], and codesigned referral pathways. Aspects of this training addressed the health needs of women of refugee background as well as improving staff understanding of refugee experiences. Midwives were also 
provided access to the online Basic Skills in Perinatal Mental Health course delivered by COPE which provides the knowledge and skills required to undertake perinatal mental health screening and use of the iCOPE digital screening platform.

Strategies developed during implementation

Staff training was delivered prior to implementation as well as ongoing access to onsite research staff with relevant knowledge regarding perinatal mental health care, EPDS administration, and use of the digital platform iCOPE for one-on-one consultancies. Existing weekly staff meetings were used to discuss process issues as they arose, facilitating a collaborative approach and shared decision-making responsibilities for managing issues, challenges, as well as noting successes.

\section{Social/professional role and identity}

Pre-implementation barriers

Health professionals identified the need for a "go-to" person within the healthcare service to support both staff and women in accessing referrals in order to improve referral uptake and service usage [18].

Pre-implementation strategies

Collaborating with the organization's refugee health service by including the manager as a member of the project steering committee enabled the mobilization of key personnel. A Refugee Health Nurse Liaison (RHNL) supported staff in the clinic and a bi-cultural worker telephoned women prior to their appointment to introduce the research project as well as asking them to attend 15 minutes earlier to complete screening.

\section{Beliefs about capabilities}

\section{Pre-implementation barriers}

The challenges of incorporating the additional task of mental health screening into routine midwifery practice was identified as a barrier. There were concerns that existing time pressures for antenatal appointments would be further exhausted by the addition of manual administration and scoring of the EPDS. There is acknowledgment that these time pressures could contribute to errors when manually calculating EPDS scores. The error rate of manual scoring for the EPDS is reported to be up to $28.9 \%$ (32). Health professionals recommended the need for a streamlined and efficient screening and referral process as well as access to a support person onsite [18].

\section{Pre-implementation strategies}

The digital screening platform, iCOPE, developed by the Centre of Perinatal Excellence (COPE) was specifically designed in order to streamline and improve the efficiency of mental health screening [31]. It allowed women to complete the screening measure on their own prior to the antenatal appointment and 
on a tablet. It takes approximately 6 - 10 minutes to complete, slightly longer if an interpreter is used. On completion, the iCOPE program automatically calculated and provided healthcare professionals with a clinical report including the overall EPDS score, response to item ten (self-harm and suicidality) and anxiety sub-scale score (based on responses to items, three, four, and five) and individual response items, eliminating scorer error. The report also included a clinical management guide to assist with discussion of results and initiate referral based on co-designed referral pathways developed for the program. The iCOPE program also generated a score based, language appropriate information for women post screening with links to further information in response to answers provided.

Strategies developed during implementation

During the implementation of the program, "organisational champions" were recognized, described as staff identified by the research team as early adopters of the screening program and quick to adapt to the implemented changes. These "organisational champions" fulfilled the role of an onsite support person and further facilitated a supportive practice environment. They were able to engage midwives who were experiencing difficulties with aspects of the implementation or showing signs of reluctance to engage.

\section{Beliefs about consequences}

Pre-implementation barriers

The stigma surrounding the disclosure of mental illness symptoms and interpreter confidentiality were both described as potential barriers to screening and accessing services [18]. Health professionals advocated for the normalisation of mental health screening and the need for a sensitive approach.

Pre-implementation strategies

During the co-design process of the screening program, stakeholders raised the importance of describing the screening program as a key component of routine pregnancy care, sensitively presenting the options of mental health services, and providing follow-up care that was perceived as useful and appropriate. The training which was delivered to all staff in the antenatal clinic included aspects of how best to introduce mental health screening, the use of suitable and non-threatening terminology, as well as reiterating that screening was part of standard and routine care for all women. Referral options included a refugee health and wellbeing service which was both multidisciplinary and culturally competent and expected to overcome some of the barriers to referral uptake.

Strategies developed during implementation

Further targeted training was deemed necessary in order to support midwives in administering item 10 on the EPDS which assesses risk of self-harm and/or suicidality. Further training was arranged with the provision flowcharts provided for clear referral pathways to manage positive responses to item 10 .

\section{Environmental context and resources}


It was unanimously agreed by health professionals that in addition to the provision of professional and trustworthy female interpreters, phone interpreters were inappropriate for mental health screening [18]. Health professionals identified the importance of translated screening measures but raised concerns over the quality of translations available for the EPDS [18]. Literal translation of the EPDS can be problematic and the best practice process of translation involves forward and back translation and testing [33].

\section{Pre-implementation strategies}

After identifying the key languages of the women attending the clinic, existing EPDS translations that had been validated were obtained and new translations were conducted using best-practice methods [33]. For one identified language, Dari, the investigation of the reliability and validity of this version formed an additional part of the program. The clinic provided face-to-face interviews with female interpreters, experienced in maternity care and perinatal mental health screening.

Barriers arising during implementation

Women with low literacy were not able to complete self-administered screening. The Burmese EPDS version was unable to load onto the digital screening platform due to issues with the script.

Strategies planned for scale-up

The provision of translation audio versions of the EPDS will allow women with low literacy, and those whose preferred language is Burmese, to have the option of self-administration $[25,26]$.

\section{Social influences}

Pre-implementation strategies

Women of refugee background often lack the social supports that most women and families rely on during pregnancy and after the arrival of a baby. Therefore, continuity of care was identified as critical for building rapport and trust in order to facilitate honest disclosure between the women and their healthcare professionals [18].

Barriers arising during implementation

Due to staffing constraints experienced in most public antenatal clinics, continuity of care can be challenging. Regular staff turnover in the clinic meant there was an ongoing need to ensure new staff received training and support to include screening as part of their routine practice.

Strategies developed during implementation

The presence of a "go-to" person such as the Refugee Health Nurse Liaison (RHNL) acted to assist staff with screening and women with referrals and follow-up. The RHNL fulfils the role of regular clinic contact 
for staff and women when there is a need to discuss mental health services and referrals. The "organizational champions" were critical in maintaining enthusiasm for the program and engaging new staff.

\section{Behavioural regulation}

Pre-implementation barriers

The health professionals identified the need for immediate follow up for positive responses to the screening questions on self-harm and suicidality [18]. Clear referral guidelines were requested in clinic rooms to ensure appropriate referrals [18].

Strategies developed during implementation

Referral pathways were co-designed by the steering committee with options for referrals dependent on EPDS scores, as well as for women of refugee or non-refugee backgrounds. This included training for the health professionals in the clinic on risk management and assessing suicidality. Clear referral pathways also allowed for improved ease of both short and long-term follow-up regarding referrals as well as improved communication between services. Stakeholder consultation identified relevant and appropriate services within the community to be included in the referral pathways. Clear and explicit flowchart diagrams were developed in order to ensure a clear process for referrals including appropriate management of self-harm and suicidality risk.

\section{Program Logic Model}

The logic model designed for the program (Figure 2) describes all of the featured elements (inputs and outputs) that were involved in implementing the perinatal mental health screening program along with the proposed short-and-long term outcomes.

\section{The Capability, Opportunity, Motivation - Behaviour Model (COM-B)}

Using the behavioural change framework of the Capability, Opportunity, Motivation, Behaviour (COM-B) model the key barriers, processes, and outcomes are described [21]. The application of this framework highlights the importance of a multi-strategy approach to behaviour change which delivers strategies that address the multiple factors at play.

\section{Implementation Guide}

The iCOPE digital platform was designed to facilitate mental health screening in healthcare settings during pregnancy by ensuring consistent and accurate interpretation of clinical scales in accordance with international clinical guidelines (31). COPE was involved in all stages of the pilot screening program from design through to delivery, including involvement in the steering committee and evaluation of the program. Based on the experiences of this pilot program as well as implementation of their digital 
screening platform across other healthcare settings, they have developed an implementation guide to assist healthcare services in implementing perinatal mental health screening (Figure 3).

\section{Discussion}

In order to identify women at risk of perinatal mental illness, there is a need to develop perinatal mental health screening programs, grounded in behaviour change theory, which can be delivered across a range of antenatal care settings and with populations with complex needs. To improve health-equity in perinatal care, the barriers to implementation must be addressed for all women, including those who experience additional barriers such as women of refugee background. This case study provided a valuable opportunity to study how a healthcare organization, in partnership with research and an NGO, could implement screening that is appropriate for populations with complex needs.

To date, routine perinatal mental health screening has been inadequately implemented creating a critical gap in maternity health care (34). The implementation of screening programs within antenatal services is often inconsistent as it requires action from different departments within the health service and across disciplines of health professionals. These findings highlight the importance of a multi-strategy approach and how successful implementation involves delivering strategies that address all of the multiple factors involved. The results from this study aim to reduce barriers to screening based on the requirements for behaviour change; capability (e.g. staff training, onsite support, clear referral pathways), opportunity (e.g. time efficient screening, self-administration, quality translations of screening measures) and motivation (e.g. consistent group of interpreters and sensitive approach to screening) (21).

This case study has a number of strengths. The antenatal clinic was chosen due to its location within one of the largest public health and teaching hospitals that services a large multicultural and refugee population. The learnings from the implementation of the screening program within this setting are generalisable to many other health service settings particularly those with diverse populations and complex needs. Ongoing stakeholder involvement facilitated co-design and an ability to evolve as challenges arose. The use of a theory informed method enabled a detailed approach to program design. Despite the many learnings this program can provide for other health services, it remains a case study and further adaption is required when implementing this approach within other settings.

\section{Conclusions}

Pregnancy is a time of increased likelihood of experiencing mental illness. Women of refugee background are at even greater risk of mental illness during this period. Therefore, mental health screening during the perinatal period is critically important in order to achieve the best health outcomes for women and their families. Implementation of perinatal mental health screening has historically been limited due to a number of health service and individual barriers. There is support for screening from both women of refugee background and health professionals with both groups acknowledging the necessity and importance $(18,25,26)$. With the provision of access to digital screening together with this 
implementation guide, informed by prior research and evaluation, health services within Australia and internationally will be encouraged to use this framework when planning their implementation.

\section{List Of Abbreviations}

COM-B Capability, Opportunity, Motivation - Behaviour Model

COPE Centre of Perinatal Excellence

EPDS Edinburgh Postnatal Depression Scale

NGO Non-Governmental Organization

NPT Normalization Process Theory

RHNL Refugee Health Nurse Liaison

TDF Theoretical Domains Framework

\section{Declarations}

\section{Ethics approval and consent to participate}

This study was performed in line with the principles of the Declaration of Helsinki. Approval was granted by the Ethics Committee of Monash University and Monash Health (14475L on 03/03/2015).

\section{Consent for publication}

Not applicable

\section{Availability of data and materials}

Data sharing is not applicable to this article as no new data were created or analyzed in this study.

\section{Competing interests}

The authors declare that they have no competing interests.

\section{Funding}

The authors received no financial support for the research, authorship, and/or publication of this article.

\section{Authors Contributions}

$\mathrm{JAB}, \mathrm{MGH}$ and KGM were responsible for study conception. All authors were involved in the design and implementation. All authors contributed to analysis and evaluation. $\mathrm{NH}$ was responsible for the 
development of the iCOPE digital screening platform. The first draft of the manuscript was written by RB and all authors commented on draft versions of the manuscript. All authors read and approved the final manuscript.

\section{Acknowledgements}

The authors would like to thank the members of the project's steering committee and the community advisory group for their invaluable guidance. We also thank staff at the antenatal clinic for their support of the project and all of the women who participated. We thank Dr. Rhonda Garad and Dr. Angela Melder from the Monash Centre for Health, Research and Implementation (MCHRI) for their expertise and review of the manuscript.

\section{Authors' Information}

RB is supported by a scholarship from Australian Rotary Health (Ian Scott PhD Scholarship), the Windermere Foundation, and the Monash Centre for Health Research and Implementation. MGH and JAB are Australian National Health and Medical Research Council Fellows.

\section{References}

1. World Health Organization. Mental health and substance abuse: maternal mental health 2021 [Available from: https://www.who.int/teams/mental-health-and-substance-use/maternal-mental-health.

2. Austin MP, Kildea S, Sullivan E. Maternal mortality and psychiatric morbidity in the perinatal period: challenges and opportunities for prevention in the Australian setting. MJA. 2007;186(7):364-7.

3. Räisänen S, Lehto SM, Nielsen HS, Gissler M, Kramer MR, Heinonen S. Risk factors for and perinatal outcomes of major depression during pregnancy: a population-based analysis during 20022010 in Finland. BMJ Open. 2014;4(11):e004883.

4. Śliwerski A, Kossakowska K, Jarecka K, Świtalska J, Bielawska-Batorowicz E. The effect of maternal depression on infant attachment: a systematic review. Int J Environ Res Public Health. 2020;17(8).

5. Austin M-P, Highett N, the Expert Working Group. Mental health care in the perinatal period: Australian clinical practice guideline 2017. [Available from: https://cope.org.au/wpcontent/uploads/2017/10/Final-COPE-Perinatal-Mental-Health-Guideline.pdf

6. Smith MV, Shao L, Howell H, Lin H, Yonkers KA. Perinatal depression and birth outcomes in a Healthy Start project. Matern Child Health J. 2011;15(3):401-9.

7. Alipour Z, Lamyian M, Hajizadeh E. Anxiety and fear of childbirth as predictors of postnatal depression in nulliparous women. Women Birth. 2012;25(3):e37-43. 
8. Pricewaterhouse Coopers. The cost of perinatal depression and anxiety in Australia 2019. [Available from: https://www.pc.gov.au/_data/assets/pdf_file/0017/250811/sub752-mental-healthattachment.pdf

9. Bauer A PM, Knapp M, lemmi V, Adelaja B. The costs of perinatal mental health problems. 2014. [Available from: https://www.nwcscnsenate.nhs.uk/files/3914/7030/1256/Costs_of_perinatal_ mh.pdf

10. Dadi AF, Miller ER, Bisetegn TA, Mwanri L. Global burden of antenatal depression and its association with adverse birth outcomes: an umbrella review. BMC Public Health. 2020;20(1):173.

11. Dennis CL, Falah-Hassani K, Shiri R. Prevalence of antenatal and postnatal anxiety: systematic review and meta-analysis. Br J Psychiatry. 2017;210(5):315-23.

12. Gavin NI, Gaynes BN, Lohr KN, Meltzer-Brody S, Gartlehner G, Swinson T. Perinatal depression: a systematic review of prevalence and incidence. Obstet Gynecol. 2005;106(5 Pt 1):1071-83.

13. Howard LM, Molyneaux E, Dennis C-L, Rochat T, Stein A, Milgrom J. Non-psychotic mental disorders in the perinatal period. Lancet. 2014;384(9956):1775-88.

14. Fellmeth G, Fazel M, Plugge E. Migration and perinatal mental health in women from low- and middle-income countries: a systematic review and meta-analysis. BJOG. 2017;124(5):742-52.

15. Reilly N, Kingston D, Loxton D, Talcevska K, Austin MP. A narrative review of studies addressing the clinical effectiveness of perinatal depression screening programs. Women Birth. 2020;33(1):51-9.

16. NICE. Antenatal and postnatal mental health: The NICE guideline on clinical management and service guidance. 2014. [Available from: https://www.nice.org.uk/guidance/cg192/resources /antenatal-and-postnatal-mental-health-clinical-management-and-service-guidance-pdf35109869806789

17. O'Connor E, Rossom RC, Henninger M, Groom HC, Burda BU. Primary care screening for and treatment of depression in pregnant and postpartum women: evidence report and systematic review for the US preventive services task force. JAMA. 2016;315(4):388-406.

18. Nithianandan N, Gibson-Helm M, McBride J, Binny A, Gray KM, East C, et al. Factors affecting implementation of perinatal mental health screening in women of refugee background. Implement Sci. 2016;11(1):150.

19. Cane J, O'Connor D, Michie S. Validation of the theoretical domains framework for use in behaviour change and implementation research. Implement Sci. 2012;7(1):37.

20. Frechtling JA. Logic modeling methods in program evaluation. San Francisco, CA, US: JosseyBass; 2007. 1-160. 
21. Michie S, van Stralen MM, West R. The behaviour change wheel: a new method for characterising and designing behaviour change interventions. Implement Sci. 2011;6:42-.

22. Pinnock H, Barwick M, Carpenter CR, Eldridge S, Grandes G, Griffiths CJ, et al. Standards for Reporting Implementation Studies (StaRI) Statement. BMJ. 2017;356:i6795.

23. Crowe S, Cresswell K, Robertson A, Huby G, Avery A, Sheikh A. The case study approach. BMC Med Res Methodol. 2011;11(1):100.

24. Boyle JA, Willey S, Blackmore R, East C, McBride J, Gray K, et al. Improving mental health in pregnancy for refugee women: protocol for the implementation and evaluation of a screening program in Melbourne, Australia. JMIR Res Protoc. 2019;8(8):e13271.

25. Willey SM, Gibson-Helm ME, Finch TL, East CE, Khan NN, Boyd LM, et al. Implementing innovative evidence-based perinatal mental health screening for women of refugee background. Women Birth. 2020;33(3):e245-e55.

26. Willey SM, Blackmore RP, Gibson-Helm ME, Ali R, Boyd LM, McBride J, et al. "If you don't ask ... you don't tell": refugee women's perspectives on perinatal mental health screening. Women Birth. 2020;33(5):e429-e37.

27. May CR, Mair F, Finch T, MacFarlane A, Dowrick C, Treweek S, et al. Development of a theory of implementation and integration: Normalization Process Theory. Implement Sci. 2009;4(1):29.

28. Greenwood-Lee J, Hawe P, Nettel-Aguirre A, Shiell A, Marshall DA. Complex intervention modelling should capture the dynamics of adaptation. BMC Med Res Methodol. 2016;16(1):51.

29. Alexander KE, Brijnath B, Mazza D. Barriers and enablers to delivery of the Healthy Kids Check: an analysis informed by the Theoretical Domains Framework and COM-B model. Implement Sci. 2014;9(1):60.

30. Cox JL, Holden JM, Sagovsky R. Detection of postnatal depression. Development of the 10-item Edinburgh Postnatal Depression Scale. Br J Psychiatry. 1987;150:782-6.

31. Centre of Perinatal Excellence. iCOPE digital screening 2019. [Available from: https://www.cope.org.au/health-professionals/icope-digital-screening/.

32. Matthey S, Matthey S, Lee C, Lee C, Črnčec R, Črnčec R, et al. Errors in scoring the Edinburgh Postnatal Depression scale. Arch Womens Ment Health. 2013;16(2):117-22.

33. World Health Organization. Process of translation and adaptation of instruments 2021 [Available from:https://www.who.int/substance_abuse/research_tools/translation/en/. 
34. Puryear LJ, Nong YH, Correa NP, Cox K, Greeley CS. Outcomes of implementing routine screening and referrals for perinatal mood disorders in an integrated multi-site pediatric and obstetric setting. Matern Child Health J. 2019;23(10):1292-8.

\section{Tables}

Table 1. Applying the COM-B model [21] to the creation of a perinatal mental health screening program 


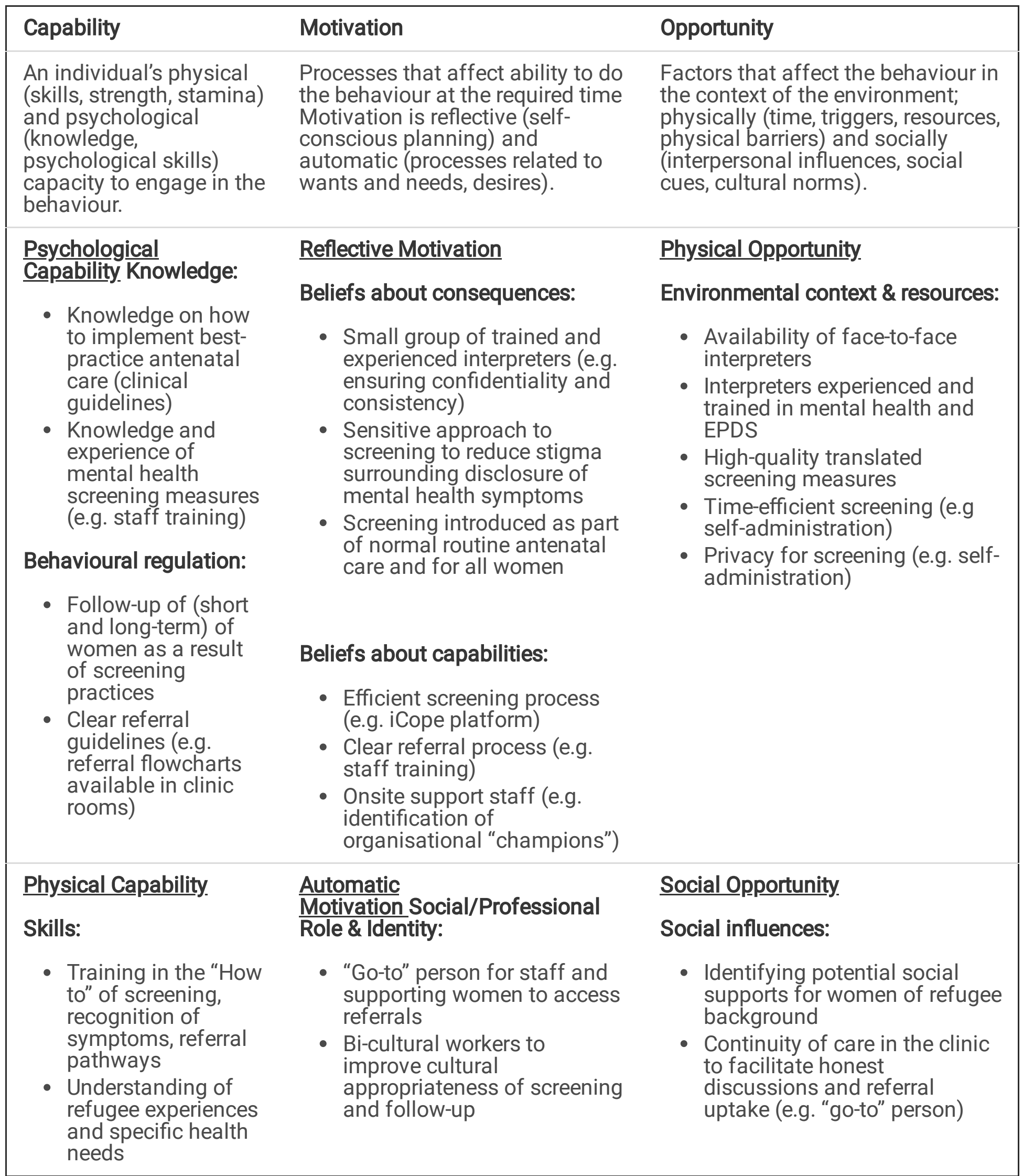

\section{Figures}




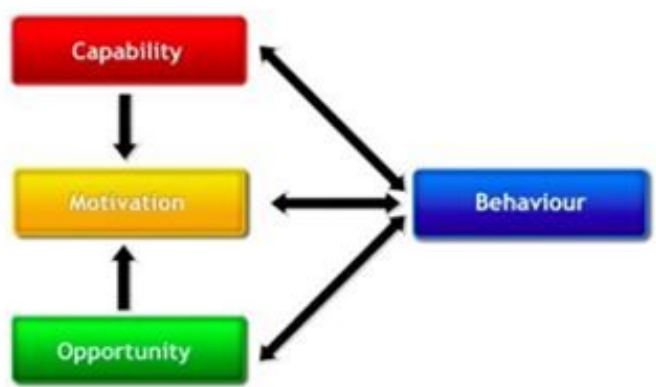

Soc - Social influences Env - Environmental Context and Resources Id - Social/Professional Role and Identity Bel Cap - Beliefs about Capabilities Opt - Optimism Int - Intentions

Goals - Goals

Bet Cons - Betiefs about Consequences Reinf - Reinforcement

Em - Emotion

Know - Knowledge

$\mathrm{Cog}$ - Cognitive and interpersonal skills Mem - Memory, Attention and Decision Processes Beh Reg - Behavioural Regulation

Phys - Physical skolts

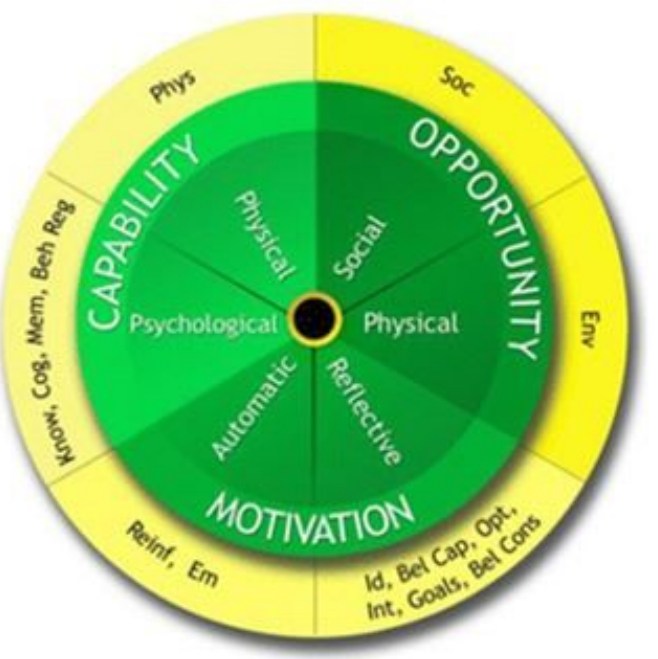

\section{Figure 1}

Map of Theoretical Domains Framework (TDF) to the COM-B model. Figure reproduced from Alexander et al. 2014 [29]. 


\section{INPUTS}

- Identification of healthcare gap

- Stakeholder consultation

- Co-development of service implementation $\&$ referral pathways

- Resource development (translation, back translation and testing)

- iPad/Tablet system of screening piloted in other populations through NGO collaboration (iCOPE)

\section{OUTPUTS}

- $\quad$ Staff training \& professional development

- Mixed methods data collection

- Translated resources

- Community partnerships and collaboration

- Increased awareness of maternal mental health

\section{OUTCOMES}

\section{Short-Term}

Increased identification of mental health symptoms

- Improved referral pathways and increased referral uptake

\section{Long-Term}

Improved health outcomes for women \& their families

Figure 2

Program Logic Model of the perinatal mental health screening program 


\section{Implementation Guide}

The iCOPE Digital Screening Program has been successfully implemented across a range of maternity settings. This brief seven-step guide is designed to help you define WHAT your program may look like and HOW to get people engaged and invested in implementing digital screening within your service.

\begin{tabular}{|c|c|}
\hline & $\begin{array}{l}\text { Start with the why } \\
\text { People need reasons to change behaviour, fund or spend time on improving clinical practice. } \\
\text { Take time to define why iCOPE digital screening is important and help people clearly } \\
\text { understand what's in it for them and their patients. They will be more excited about helping. }\end{array}$ \\
\hline & $\begin{array}{l}\text { Mobilise the right leadership } \\
\text { Implementation is a social process that relies on people to drive commitment. } \\
\text { Enlist support from influential leaders from key disciplines who will guide, make decisions, } \\
\text { learn, share, actively problem solve, and stay inwolved to make the iCOPE happen. }\end{array}$ \\
\hline & $\begin{array}{l}\text { Take a collaborative, interdisciplinary approach } \\
\text { People are more likely to support what they heip create. } \\
\text { Invite representatives likely to be impacted by iCOPE screening (e.g. midwives, allied health, } \\
\text { administrative staff, medical records and IT teams) to be involved. Enlist their ideas and help } \\
\text { them to understand how iCOPE screening impacts them and how you can work together. }\end{array}$ \\
\hline & $\begin{array}{l}\text { Define your Clinical Program } \\
\text { Each setting is different. } \\
\text { iCOPE can be tailored to support your setting, clinicians and patients. Our team will support as } \\
\text { you plan to implement iCOPE into your screening schedules, patient cohorts, record keeping } \\
\text { systems, communication channels and and referral pathways. }\end{array}$ \\
\hline & $\begin{array}{l}\text { Do the 'techy' Stuff } \\
\text { While implementing iCOPE is not difficult, optimal outcomes are achieved with IT integration. } \\
\text { If this is your goal, our team will work closely with your IT representatives to enable clinical } \\
\text { reports to be seamlessly integrated into existing electronic health record systems. }\end{array}$ \\
\hline & $\begin{array}{l}\text { Prepare people } \\
\text { To achieve lasting change in clinical practice, consistency is key. } \\
\text { Once you've raised awareness and desire to participate, help teams get ready by equipping } \\
\text { them with the knowledge, skills, support tools and mentoring to help them confidently deliver } \\
\text { iCOPE screening. We will assist in the provision of learning materials to prepare your people. }\end{array}$ \\
\hline & $\begin{array}{l}\text { Monitor, Evaluate } 8 \text { Improve } \\
\text { Ongoing evaluation is an integral part of any quality care initiative. } \\
\text { It helps clearly define your desired outcomes at the outset, and decide how and when you'll } \\
\text { measure success. By collecting baseline data of your current practice and then continuing to } \\
\text { monitor results, you can assess and adjust your program as needed. }\end{array}$ \\
\hline
\end{tabular}

The Prime Minister's commitment to a Perinatal Mental Health Check.

o Centre of Perinatal Excellence 2020 (version 1.0_260710)

Further information regarding each of these steps can be found at: https://www.cope.org.au/hospital-to-home/

\section{Figure 3}

COPE implementation guide for perinatal mental health screening

\section{Supplementary Files}

This is a list of supplementary files associated with this preprint. Click to download. 
- StaRIchecklist.docx

Page 21/21 